\title{
latrogenic Arteriovenous Fistula Secondary to Coronary Artery Dissection Caused by Contrast Injection Into a Chronically Occluded Right Coronary Artery
}

\author{
Benjamin Fogelson ${ }^{\mathrm{a}}$, Hassan Tahir ${ }^{\mathrm{a}}$, James Livesay ${ }^{\mathrm{a}}$, Raj Baljepally ${ }^{\mathrm{a}, \mathrm{b}}$
}

\begin{abstract}
Coronary arteriovenous fistulas and coronary cameral fistulas are rare anomalies that involve the abnormal communication between a coronary artery and a venous structure, such as a coronary vein or a right-sided cardiac chamber. Iatrogenic coronary arteriovenous fistulas and coronary cameral fistulas can be uncommon complications of coronary artery angiography and intervention. Acquired coronary arteriovenous fistulas that develop during percutaneous coronary intervention of chronic total occlusions have been previously reported in the literature. However, a coronary arteriovenous fistula resulting from contrast injection into a chronically and totally occluded right coronary artery during diagnostic coronary angiography is very rare. We present a unique case of a contrast-induced iatrogenic right coronary dissection leading to a coronary arteriovenous fistula communicating to the right atrium through the middle cardiac vein.
\end{abstract}

Keywords: Coronary arteriovenous fistula; Coronary cameral fistula; Chronic total occlusion; Coronary artery dissection

\section{Introduction}

Coronary arteriovenous fistula (CAVF) is a rare anomaly that involves the abnormal communication between a coronary artery and an adjacent venous structure, most commonly, a coronary vein $[1,2]$. Fistulas can also develop between the coronary arteries and other cardiac structures such as the cardiac atria or ventricles, which is known as a coronary cameral fistula (CCF) $[2,3]$. Patients with CAVF are usually asymptomatic; however patients with significant left to right shunting can develop symptoms such as shortness of breath, chest pain, and

Manuscript submitted July 26, 2021, accepted August 10, 2021

Published online September 29, 2021

aDepartment of Medicine, University of Tennessee Graduate School of Medicine, Knoxville, TN, USA

${ }^{b}$ Corresponding Author: Raj Baljepally, University of Tennessee Medical Center in Knoxville, 1924 Alcoa Hwy, Knoxville, TN 37920, USA.

Email: RBaljepa@utmck.edu

doi: https://doi.org/10.14740/jmc3754 fatigue. Rarely, CAVF can cause myocardial infarctions and arrhythmias due to diminished flow within the coronary artery distal to the fistula [1]. The etiology of CAVF and CCF can be congenital or acquired. The main causes of acquired CAVF include myocardial infarction, chest trauma, coronary artery aneurysm or dissection, coronary angioplasty, and coronary artery bypass grafting $(\mathrm{CABG})[2,4,5]$. Although rare, CAVF is a known complication of percutaneous coronary intervention (PCI) $[6,7]$. CAVF caused by coronary dissection due to contrast injection during diagnostic angiography is a very rare complication. We present a unique case of an iatrogenic CAVF secondary to a right coronary artery (RCA) chronic total occlusion (CTO) dissection caused by contrast injection during a diagnostic coronary angiogram.

\section{Case Report}

\section{Investigations}

A 72-year-old female presented to the emergency department with chest pain and shortness of breath. Her history was significant for prior CABG surgery with a left internal mammary artery (LIMA) to the left anterior descending (LAD) artery, a saphenous vein graft (SVG) to the obtuse marginal artery, and an SVG to the RCA. On initial workup the patient was found to have a non-ST elevation myocardial infarction with an elevation in cardiac enzymes and nonspecific ST-segment changes on electrocardiogram. We proceeded with a left heart catheterization and coronary angiogram.

\section{Diagnosis}

The patient's coronary angiography revealed $90 \%$ stenosis in the proximal SVG to RCA. Her angiogram also demonstrated a CTO of the native RCA, which was previously known based on prior coronary angiograms (Supplementary Material 1, www. journalmc.org). During the initial contrast injection, where 7 - 8 $\mathrm{mL}$ of contrast was injected at $3 \mathrm{~mL} / \mathrm{s}$ into the native RCA, a dissection occurred at the location of the CTO (Fig. 1 and Supplementary Material 2, www.journalmc.org). Unfortunately, the development of the dissection was unnoticed, and a second $7-8$ $\mathrm{mL}$ of contrast was administered at $3 \mathrm{~mL} / \mathrm{s}$ into the RCA. The 


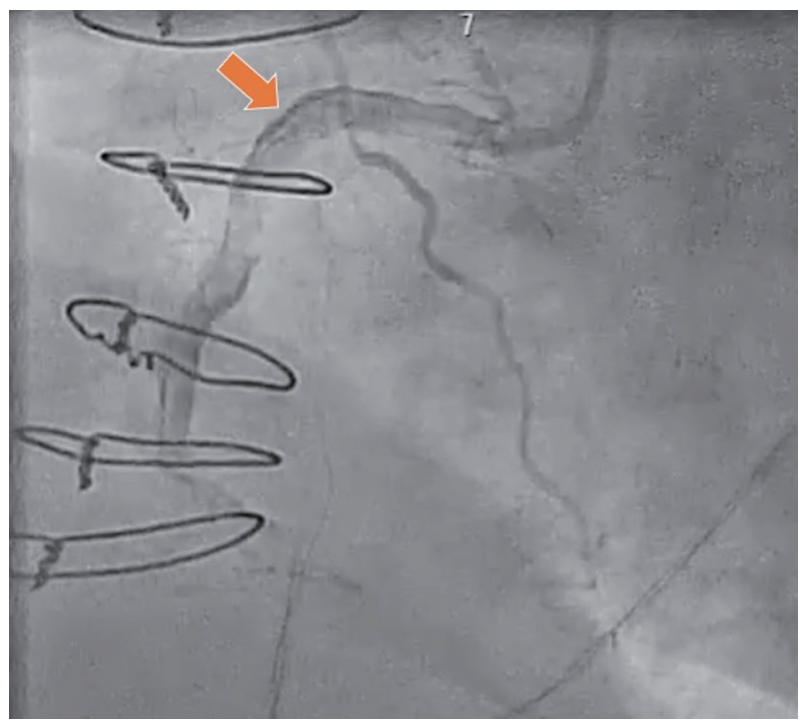

Figure 1. Contrast injection into the right coronary artery causing dissection at the location of the chronic total occlusion that progressed distally. Arrow indicates contrast within the right coronary artery dissection.

second contrast injection into the RCA resulted in progression of the coronary dissection causing a CAVF with the middle cardiac vein distally (Fig. 2 and Supplementary Material 3, www.journalmc.org). Additionally, there was a right coronary cusp dissection proximally (Fig. 2 and Supplementary Material 3, www. journalmc.org). Initially, there was concern that the second contrast injection had caused a perforation of the mid segment of the RCA. However, upon further review of the angiogram images, contrast could be seen flowing distally into the middle cardiac vein and emptying into the right atrium (Fig. 2 and Supplementary Material 3, www.journalmc.org).

\section{Treatment}

The decision was made to manage the patient conservatively as we believed the CAVF would spontaneously close within 4 - 8 weeks as the RCA CTO dissection healed. She was closely monitored following her procedure in the cardiovascular unit. Two days after her initial coronary angiogram, she successfully underwent PCI of the SVG to RCA. The presence of the CAVF did not interfere with the PCI of the SVG to RCA.

\section{Follow-up and outcomes}

Following successful PCI of the SVG to RCA, the patient was observed overnight in the cardiac observation unit. She had no complications following her procedure and was therefore safely discharged home in stable condition. She is regularly seen in our clinic and has not reported any new concerns or adverse events following the initial hospitalization. Repeat angiography of the native RCA to evaluate for fistula closure was deferred due to the risk of worsening or repeat dissection and/or fistula.

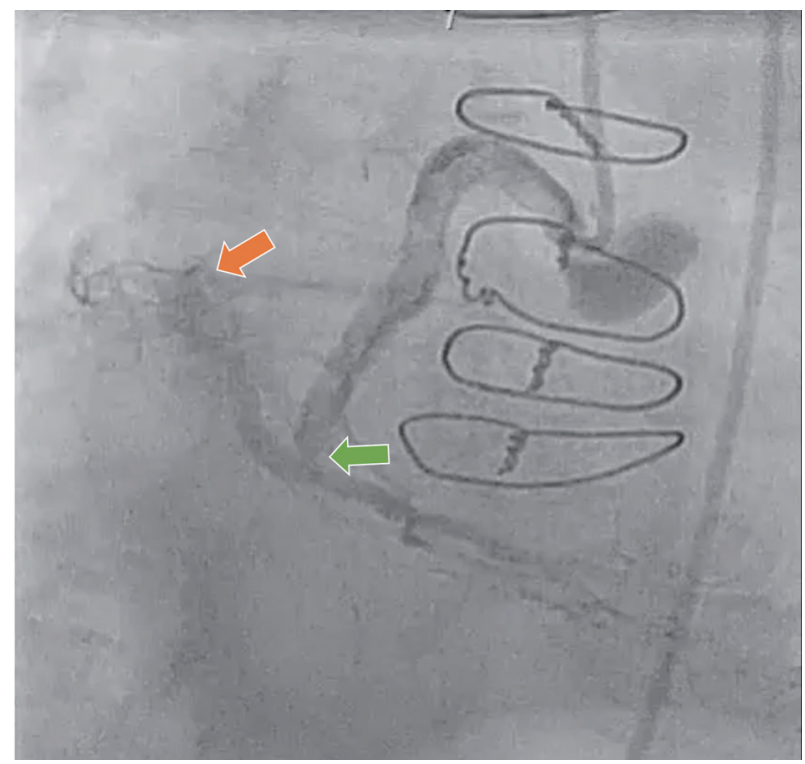

Figure 2. Subsequent contrast injection into the right coronary artery leading to progression of the coronary dissection distally and the development of a right coronary artery-to-middle cardiac vein fistula (green arrow) which empties into the right atrium (orange arrow).

\section{Discussion}

The development of CAVF is a rare, but challenging, complication of diagnostic coronary catheterization and PCI. The overall incidence of CAVF, including both congenital and acquired causes, is less than 1\% [1]. Iatrogenic CAVF most commonly involves the RCA followed by the LAD and circumflex artery, respectively [1]. The typical sites of CAVF drainage are the right atrium, right ventricle, superior vena cava, and coronary sinus due to their relatively low pressures when compared to the left atrium and left ventricle [1]. The clinical manifestations of CAVF vary depending on the etiology of the fistula and the severity of left to right shunting; however, the majority of CAVF in adults are asymptomatic [1].

There have been prior published case reports of iatrogenic CAVF and CCF that developed following various coronary artery interventions (Table 1 [2, 6-9]). The more common causes of iatrogenic CAVF and CCF include coronary perforation during stenting and guidewire-induced trauma $[6,8]$. Ohayon et al reported the development of a LAD-toleft ventricle fistula following primary PCI for an anterior ST-elevation myocardial infarction [6]. Additionally, recent technique advancements have allowed for an increase in the number of performed CTO interventions. Consequently, the incidence of coronary artery complications has increased in proportion with the number of CTO interventions [7, 9]. According to Kobayashi et al, the development of CAVF from the septal artery into the right ventricle is a rare complication of the retrograde CTO PCI approach [9]. Similarly, Park et al reported a case of a guidewire-induced CAVF between the left circumflex artery and the posterior vein of the left ventricle that occurred during CTO PCI [7]. Narasimhan et al presented a CAVF that developed between the circumflex artery and the 
Table 1. Prior Published Case Reports of latrogenic CAVF and CCF Including Location and Etiology of the Fistula

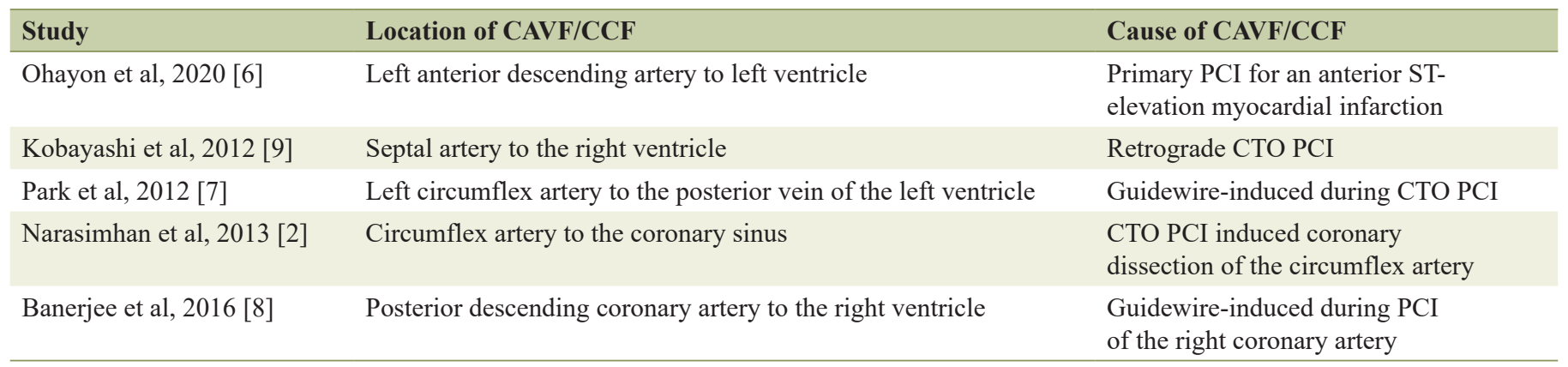

CAVF: coronary arteriovenous fistula; CCF: coronary cameral fistula; PCl: percutaneous coronary intervention; CTO: chronic total occlusion.

coronary sinus secondary to a CTO PCI induced coronary dissection of the circumflex artery [2]. Almost all cases of CTO related CAVF were secondary to CTO PCI. We report an iatrogenic CAVF between the RCA and middle cardiac vein that developed due to a coronary artery dissection secondary to contrast injection into a CTO during diagnostic coronary angiography, which then resulted in a CCF to the right atrium through a communication with the middle cardiac vein. Our case is unique and to our knowledge, has not been previously reported.

Due to their rarity, the management of CAVF and CCF is debated amongst many experts. The majority of CAVF and CCF close spontaneously and are usually managed conservatively $[1,4]$. According to Gowda et al, invasive treatments for CAVF are primarily reserved for patients with symptomatic fistulas and those at risk for future complications [1]. Invasive treatment modalities for CAVF include surgical correction, stenting, and embolization. Park et al demonstrated the use of a covered stent to repair an iatrogenic left circumflex coronary artery-to-posterior vein of the left ventricle fistula that developed during CTO PCI [7]. Banerjee et al reported the successful use of coil embolization to treat a guidewireinduced posterior descending coronary artery-to-right ventricle fistula that developed secondary to PCI [8]. In our case, the decision was made to proceed with conservative management with close observation.

\section{Learning points}

CAVFs can rarely occur secondary to complex coronary interventions such as CTO PCI. However, diagnostic coronary angiography causing a CAVF is extremely rare. Contrast injection into a chronically occluded vessel during diagnostic coronary angiography can lead to coronary dissection and CAVF. Our case highlights the importance of cautious contrast injection of any chronically occluded vessel to prevent this rare complication.

\section{Supplementary Material}

Suppl 1. Previous coronary angiogram shows chronic total oc- clusion (CTO) of proximal right coronary artery (RCA).

Suppl 2. Contrast injection into the right coronary artery causing a dissection starting at the location of the chronic total occlusion and progressing distally.

Suppl 3. Subsequent contrast injection into the right coronary artery leading to progression of the coronary dissection distally and the development of a right coronary artery-to-middle cardiac vein fistula which empties into the right atrium.

\section{Acknowledgments}

None to declare.

\section{Financial Disclosure}

None to declare.

\section{Conflict of Interest}

None to declare.

\section{Informed Consent}

The informed consent was obtained.

\section{Author Contributions}

All authors reviewed the literature and helped write the manuscript. Benjamin Fogelson, DO, Hassan Tahir, MD, James Livesay, DO, and Raj Baljepally, MD performed critical revisions of article and approved the final version of manuscript.

\section{Data Availability}

The authors declare that data supporting the findings of this study are available within the article. 


\section{References}

1. Gowda RM, Vasavada BC, Khan IA. Coronary artery fistulas: clinical and therapeutic considerations. Int J Cardiol. 2006;107(1):7-10.

2. Narasimhan S. Coronary arteriovenous fistula secondary to percutaneous coronary intervention of chronic total occlusion. Case Rep Vasc Med. 2013;2013:706820.

3. Minhas AM, Ul Haq E, Awan AA, Khan AA, Qureshi G, Balakrishna P. Coronary-cameral fistula connecting the left anterior descending artery and the first obtuse marginal artery to the left ventricle: a rare finding. Case Rep Cardiol. 2017;2017:8071281.

4. Schanzenbacher P, Bauersachs J. Acquired right coronary artery fistula draining to the right ventricle: angiographic documentation of first appearance following reperfusion after acute myocardial infarction, with subsequent spontaneous closure. Heart. 2003;89(8):e22.
5. Mehran R, Claessen BE, Godino C, Dangas GD, Obunai K, Kanwal S, Carlino M, et al. Long-term outcome of percutaneous coronary intervention for chronic total occlusions. JACC Cardiovasc Interv. 2011;4(9):952-961.

6. Ohayon P, Matta A, Boudou N. A case report of an iatrogenic coronary cameral fistula treated by retrograde percutaneous coronary intervention. Eur Heart J Case Rep. 2020;4(3):1-6.

7. Park SH, Rha SW, Cho AR, Lee HG, Choi CU, Oh DJ. Successful management of iatrogenic coronary arteriovenous fistula developed during chronic total occlusion intervention. J Cardiol Cases. 2012;6(4):e96-e99.

8. Banerjee S, Patra S. Coronary-cameral fistula caused by guidewire trauma and resolved by coil embolization. Tex Heart Inst J. 2016;43(4):338-340.

9. Kobayashi Y. Guidewire-induced iatrogenic coronary arteriovenous fistula: An accidental meeting. J Cardiol Cases. 2012;6(4):e124-e125. 